\title{
Leaf Doctor: A New Portable Application for Quantifying Plant Disease Severity
}

Sarah J. Pethybridge, School of Integrative Plant Science, Section of Plant Pathology and Plant-Microbe Biology, Cornell University, Geneva, NY 14456; and Scot C. Nelson, College of Tropical Agriculture and Human Resources, Department of Plant and Environmental Protection Sciences, University of Hawaii at Manoa, Honolulu, HI 96822

\begin{abstract}
Pethybridge, S. J., and Nelson, S. C. 2015. Leaf Doctor: A new portable application for quantifying plant disease severity. Plant Dis. 99:1310-1316.

An interactive, iterative smartphone application was used on color images to distinguish diseased from healthy plant tissues and calculate percentage of disease severity. The user touches the application's display screen to select up to eight different colors that represent healthy tissues. The user then moves a threshold slider until only the symptomatic tissues have been transformed into a blue hue. The pixelated image is then analyzed to calculate the disease percentage. This study reports the accuracy, precision, and robustness of Leaf Doctor using six different diseases with typical lesions of varying severity. Estimates of disease severity from Leaf Doctor were highly accurate $\left(R^{2} \geq 0.79 ; C_{\mathrm{b}} \geq 0.959\right)$ compared with estimates obtained from the discipline-standard, Assess. Precision

was operationally defined as the ability of a rater to use Leaf Doctor and repeatedly obtain similar percentages of disease severity for the same image. Coefficients of variation were low $(0.51$ to $14.1 \%)$ across all disease datasets but a significant negative relationship was found between the coefficient of variation of estimates and mean disease severity. Other advantages of Leaf Doctor included comparatively less time for image processing, low cost, ease of use, ability to send results by e-mail, and the ability to create realistic standard area diagrams. Leaf Doctor is compatible with iPhone, iPad, and iPod touch and is optimized for iPhone 5. It is available as a free download at the iTunes Store.
\end{abstract}

Reliable, accurate assessments of disease intensity are critical for many research areas in plant pathology, including evaluations of cultural and pesticidal management practices, spatiotemporal modeling of epidemics, crop loss models, dispersal gradients, disease forecasting, understanding relationships between symptoms and the environment, and evaluating germplasm for resistance to pathogens (Gaunt 1995; Kranz 1988). Despite the importance of ensuring that phytopathometric assessments are both accurate and precise, these ratings are typically derived from visual assessments at various levels of resolution (plots, plants, and/or tissues). A common approach to quantifying disease intensity is to estimate its severity, defined here as the amount (e.g., area) of diseased plant tissue relative to the total amount of susceptible tissue available (Nutter et al. 1991). In addition, severity may be expressed as the number of lesions per sampling unit for rusts and other diseases. Disease incidence, defined as the diseased proportion of a population's sampling units (plant, organs) can also indicate disease intensity (Nutter et al. 1991). Incidence and other count data are inherently less subject to bias and errors of perception by raters, as compared with visual estimates of disease severity.

The potential pitfalls of assessing disease severity visually, using either a single (intra) rater or a team (inter) of raters, are numerous and have been well documented (Nilsson 1995; Nutter et al. 1993). These drawbacks include rater fatigue and the decrease in accuracy and precision of rater estimates over time due to the repetitive, physically tiring nature of assessment tasks. Furthermore, individual raters can return highly divergent values in their visual assessments of the same sampling unit (Nutter et al. 1993). The use of standard area diagrams as assessment tools substantially improves the accuracy, precision, and reliability of intra- and interrater estimates of plant disease severity (Bardsley and Ngugi 2013; Bock et al. 2008; Braido et al. 2014; James 1971; Price et al. 1993; Sherwood et al.

\section{Corresponding author: S. C. Nelson; E-mail: snelson@ hawaii.edu}

*The $\boldsymbol{e}$-Xtra logo stands for "electronic extra" and indicates that 1 supplementary figure is included in the online edition.

Accepted for publication 16 April 2015.

http://dx.doi.org/10.1094/PDIS-03-15-0319-RE

(C) 2015 The American Phytopathological Society
1983; Spolti et al. 2011). Nutter and Schultz (1995) reported that using a computerized disease assessment training program significantly improved the ability of trainees to accurately estimate disease severity. The raters completed at least $1 \mathrm{~h}$ of training, during which they provided their best estimates and received instant feedback on the true disease severity of the sample. Hence, raters became immediately aware of their bias and able to adjust their approach in training and not when sampling during field experiments.

Along with advances in computer training and assessment algorithms, improvements in automation processes for phytopathometry have been made that reduce subjective intra- and interrater errors. Many of these computer programs are now packaged into commercially available, proprietary software. The most commonly used semiautomatic computer program to estimate disease intensity is Assess (Lamari 2002). However, with the advent of open-source software for image analysis via freeware platforms such as ImageJ (Abramoff et al. 2004), or proprietary platforms like SigmaScan Pro (Systat Software, San Jose, CA) and Adobe Photoshop (Adobe Systems Incorporated, San Jose, CA), users can construct macros for processing specific tasks in image analysis (Wijekoon et al. 2008). This macro approach requires a user to possess sufficient computer programming skills and the time to write distinct macros for different diseases. Subsequently, image analysis programs have been designed specifically to support plant pathology research objectives, but many have not been evaluated beyond the diseases for which they were designed (Kwack et al. 2005; Lindow and Webb 1983; Patil and Bodhe 2011). A smaller number have been designed as semiautomatic or automatic platforms to measure disease severity across pathosystems (Barbedo 2014; Lamari 2002; Tucker and Chakraborty 1997).

The objectives of this study were i) to develop and evaluate a simple, interactive smartphone application for quantitative assessment of plant disease intensity and ii) to quantify the accuracy, precision, and robustness of the application compared with Assess, a commonly used, semiautomatic computer program for measuring disease severity on leaves.

\section{Materials and Methods}

Background, Leaf Doctor function, algorithm, and operation. Instructions for using Leaf Doctor are provided on the "About" screen of the app. The assessment algorithm depends on sequential, user-guided touching of a distinct series of recognized pixel areas in the image that correspond to healthy plant tissues (e.g., shades of green). Users may collect images for analysis in real time using an 
iPhone camera, or retrieve images already saved. Leaves or other plant organs are photographed individually against a black, nonreflective backdrop or background (Fig. 1A). Alternatively, a photograph may be modified using image editing software to create a black background that completely surrounds the assessment area

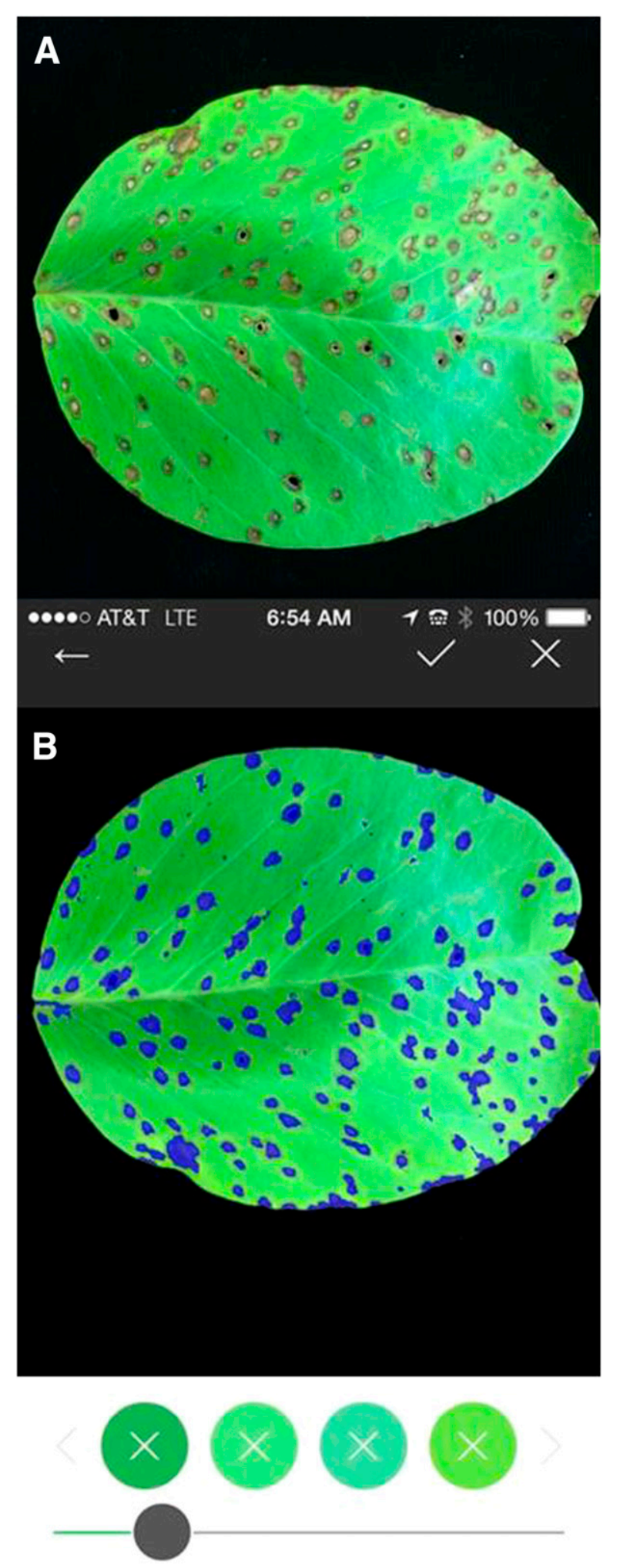

Threshold

Fig. 1. Cercospora leaf spot (Cercospora alabamensis) on beach morning-glory (A). Using Leaf Doctor, the user selects four colors on the leaf surface to represent healthy tissue (green circles), and then uses the threshold slider (gray circle) to define the maximum distance between a healthy and diseased leaf pixel, creating blue-colored lesions (B). of interest (e.g., a leaf). The image may then be e-mailed to the user to save in the phone's gallery collection for subsequent analysis by Leaf Doctor. In the field, images should be taken in shade, such as that provided by an umbrella or dense plant canopy, and preferably without flash. This helps to minimize reflected light from leaf surfaces, which can alter the natural colors of the cuticle and complicate the disease measurement algorithm. Reflected light can be problematic for image analysis on leaves with thick or shiny cuticles as it reduces the ability of a user to differentiate colors associated with diseased and healthy areas on the leaves.

To begin a disease assessment, the user touches a healthy area of the leaf on the photograph. This brings up a circular magnifying lens that is activated while the user is touching the image and specifies a distinct color that corresponds with healthy tissues. The central area of the circular magnifying lens can precisely select various colors, such as narrow leaf veins. The algorithm can use values specified by the user for up to eight colors of healthy tissue per image. The algorithm then evaluates the color of each pixel in the image for its proximity to the specified healthy colors and assigns a status of either healthy or diseased. The maximum distance from a healthy color can be adjusted by using the threshold slider bar. When the user is satisfied that the diseased tissues are represented accurately and completely (i.e., colored blue), they are ready to calculate the disease percentage (Fig. 1B). If unsatisfied, the user may improve accuracy by touching the screen to remove or add additional colors for healthy tissues. The assessment data and accompanying images may be sent by e-mail to any recipients (Fig. 2). Metadata may also be added to the body of the e-mail as text to supplement the data, and include names of the host and disease, location, or experimental treatment.
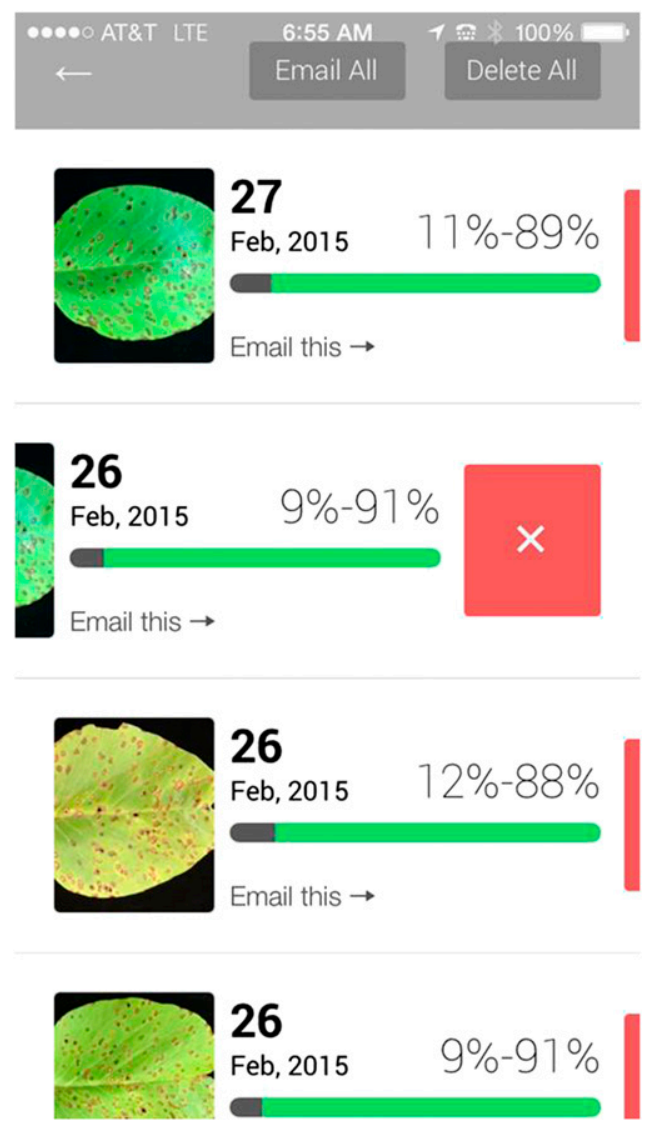

Fig. 2. Severity of Cercospora leaf spot (Cercospora alabamensis) on beach morningglory summarized by Leaf Doctor. Percentages represent the Leaf Doctor's assessment of diseased and healthy areas of the leaf surfaces. Results may be e-mailed and include metadata, such as the names of the host, disease, location, or treatment. Data may be deleted by sliding the individual cell to the left to reveal the red delete box. 
Table 1. Characteristics of disease images used to test the precision and accuracy of Leaf Doctor $(n=50$ individual leaves/disease)

\begin{tabular}{|c|c|c|c|c|c|}
\hline Disease (host) & Pathogen & Disease lesion description & $\begin{array}{l}\text { Host leaf shape } \\
\text { (margin) }\end{array}$ & $\begin{array}{l}\text { Minimum disease } \\
\text { intensity }(\%)\end{array}$ & $\begin{array}{c}\text { Maximum disease } \\
\text { intensity }(\%)\end{array}$ \\
\hline Tan spot (lima bean) & Boeremia exigua var. exigua & $\begin{array}{l}\text { Necrosis, variable lesion } \\
\text { size and color }\end{array}$ & Oval (entire) & 0.29 & 35.42 \\
\hline Downy mildew (collards) & Peronospora parasitica & Chlorosis & Oval (entire) & 0.59 & 67.38 \\
\hline Rust (mallow) & Puccinia malvacearum & Pustules (abaxial) & Circular (crenate) & 0.25 & 46.63 \\
\hline Powdery mildew (lilac) & Microsphaera alni & Mycelia & Oval (entire) & 14.21 & 93.19 \\
\hline Foliar disease complex (tomato) & $\begin{array}{l}\text { Alternaria solani }+ \\
\text { Septoria lycopersici }\end{array}$ & Necrosis and chlorosis & Variable (lobed) & 4.18 & 83.01 \\
\hline Gummy stem blight (watermelon) & Didymella bryoniae & Necrosis & Variable (lobed) & 0.72 & 87.57 \\
\hline
\end{tabular}

A

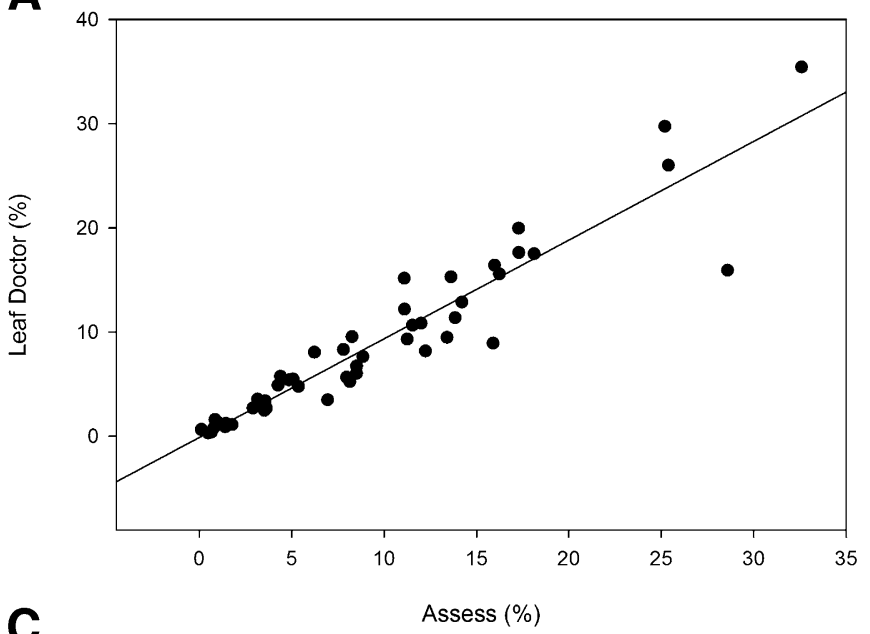

C

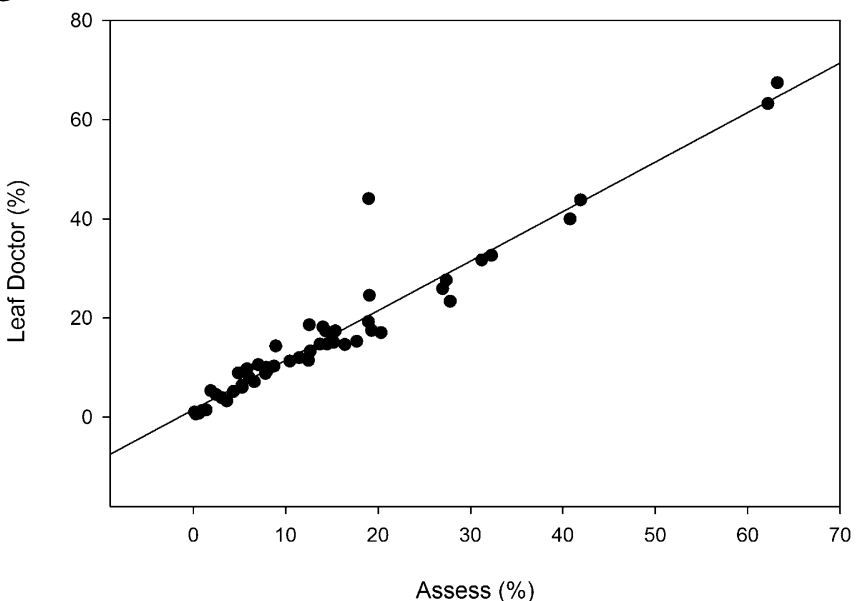

E

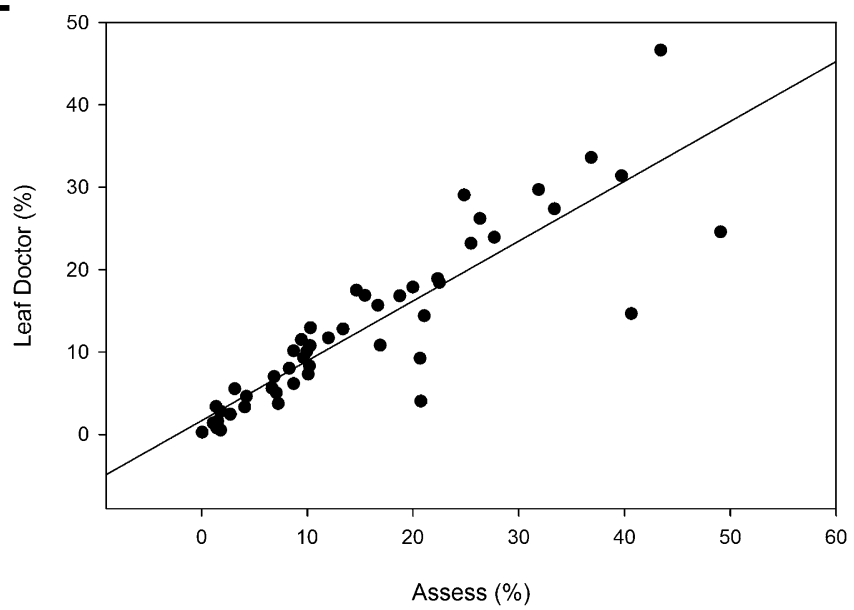

B
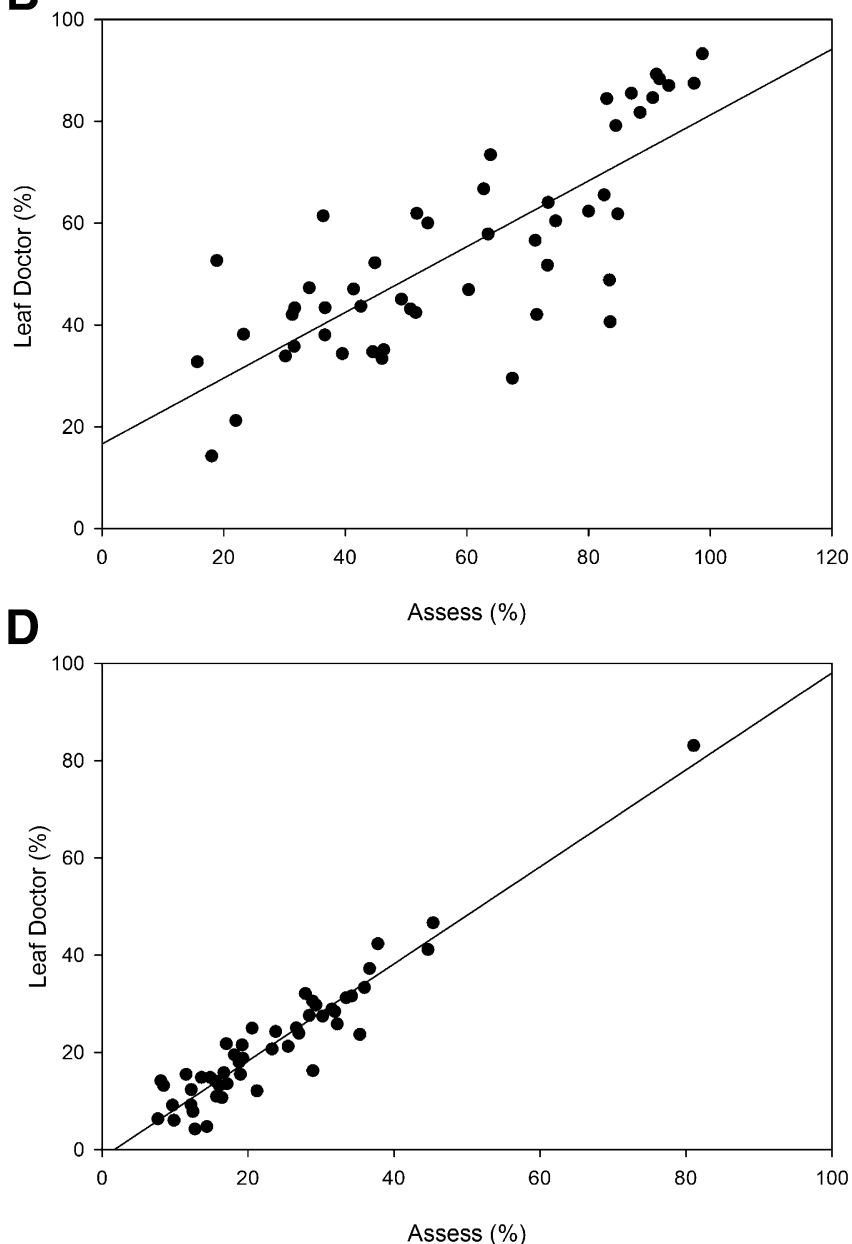

$\mathbf{F}$

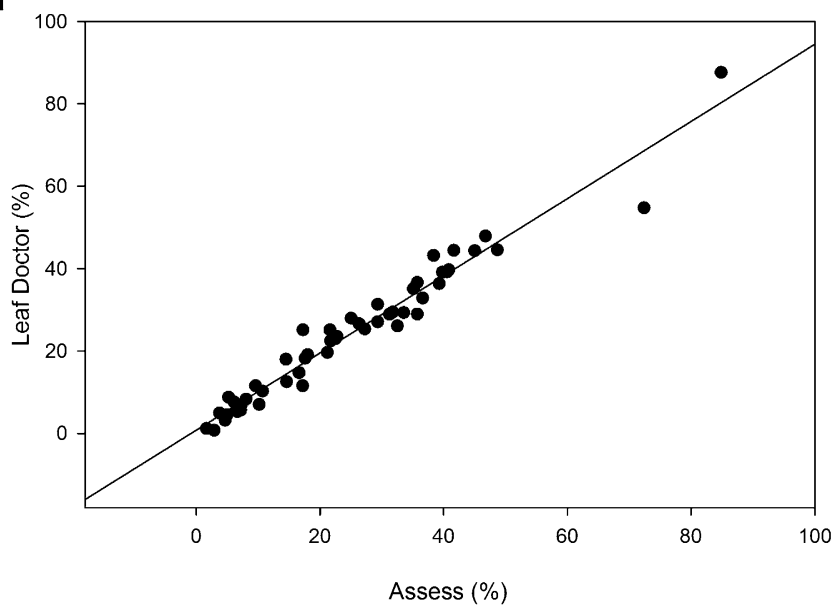

Fig. 3. Linear relationships between disease severity estimates from Assess and Leaf Doctor for 50 diseased leaves collected from each of six epidemics in the field: tan spot of lima bean $(\mathbf{A})$, downy mildew of collards (B), mallow rust $(\mathbf{C})$, lilac powdery mildew (D), a foliar disease complex in tomato (E), and gummy stem blight of watermelon (F). 
Disease images. Databases for six different plant diseases from symptomatic samples collected in the field were compiled. The disease samples represented a range of lesion types in order to evaluate the robustness of the analytic algorithms for Leaf Doctor and Assess. Each database consisted of 50 individual leaves from each pathosystem, representing a broad range of disease severity (Table 1; Supplementary Figure S1). Each leaf was photographed using an iPad's 8-megapixel camera with $f / 2.4$ aperture positioned $30 \mathrm{~cm}$ above each specimen. No camera flash was used and the specimens were placed on minimally reflective, black velvet cloth. Lights only directly above the sampling units were extinguished to minimize reflected light. Minor edits for each image were made before analysis using Adobe Photoshop Elements, Version 12.1 (San Jose, CA). Adjustments were typically for exposure and color, to sharpen the image using the Auto Smart Fix function, or to correct inconsistencies or hue anomalies within the background.

Image analysis. Leaf Doctor was downloaded from iTunes to an iPad (https://itunes.apple.com/us/app/leaf-doctor/id874509900? $1 \mathrm{~s}=1 \& \mathrm{mt}=8)$. The images from each of the six disease databases previously edited in Photoshop were then imported individually into the application and processed as generally described above. The processed images were saved and the analytical results for each image recorded for statistical analysis.

Leaf Doctor versus Assess. Disease severity estimates from Leaf Doctor were compared with Assess Version 2.0 (Lamari 2002). For validation purposes, Assess was regarded as the discipline-standard, semiautomatic image analysis system. The same edited images were also imported individually into Assess and the automatic mode of analysis used to quantify disease severity. The disease severity estimate for each image from Assess was considered the discipline standard for quantifying the accuracy of those acquired through Leaf Doctor. Robustness of the two methods was determined by comparing estimates derived for each of the six different diseases (Table 1). Linear regression analyses modeled the relationship between disease severity assessments obtained for each image through Assess (independent variable; $x$ ) and Leaf Doctor (dependent variable; $y$ ). Accuracy was therefore operationally defined as the degree of closeness of measured values using Leaf Doctor to the estimates provided by Assess (Madden et al. 2007). Variables used to summarize these relationships were: slope, $y$-intercept, coefficient of determination $\left(R^{2}\right)$, and the standard error of the estimate for $y$. Estimates of slope and $y$-intercepts were tested for their significance from 1 and 0 , respectively. A concordance correlation coefficient $\left(C_{b}\right)$ was also calculated to assess concordance between estimates obtained through Leaf Doctor and Assess (Lin 1989). All analyses were conducted through Genstat Version 17; VSN International, Hemel Hempstead, United Kingdom.

Precision was defined as the repeatability or variation associated with individual rater estimates of disease severity on the same image using Leaf Doctor. To quantify precision, 10 edited images from each disease dataset were randomly selected. Disease severity for these images was quantified separately for five replications. Disease severity for each replication was recorded and the respective means and standard deviations calculated for each disease and image combination. This information was used to calculate coefficients of variation within each disease dataset. Relationships between coefficients of variation and mean disease severity were described by linear or nonlinear regression analyses (Genstat Version 17).

To indicate time of image processing and ease of program use, the total numbers of seconds taken to process 10 randomly selected images for each disease dataset using both Assess and Leaf Doctor were recorded. Results were summarized by calculating the average and standard deviation for times used to process images by each method.

Creation of a standard area diagram using Leaf Doctor. A standard area diagram was constructed from images processed by Leaf Doctor for Cercospora leaf spot of beach morning-glory (Ipomoea pes-caprae subsp. brasiliensis), caused by Cercospora alabamensis G. F. Atk. (Stevens 1925). Leaf samples were collected from a landscape planting at the University of Hawaii at Manoa campus,
Honolulu, HI, and assessed for disease severity during 2014. A complete range of diseased leaves with disease severity values up to the maximum observed were collected over a 6-month period. A standard area diagram with six disease categories, plus one for zero disease, was created to reflect the range of disease severity values up to the maximum. Categories were established logarithmically according to the Horsfall-Barratt rating scale (Horsfall and Barratt 1945). Images were selected to approximate the midpoint of the range for each assessment category. The total time needed to collect the required number of leaf samples $(n=27)$, photograph, quantify disease severity using Leaf Doctor, and to create the standard area diagram was recorded.

\section{Results}

Leaf Doctor versus Assess. Accuracy. Estimates of disease severity by Leaf Doctor and Assess for the same images were highly similar with significant $(P<0.001)$ linear relationships (Fig. 3; Table 2). Coefficients of variation were less than $3 \%$, indicating high precision. The amount of variation in Leaf Doctor estimates explained by Assess were at least 0.94 for the diseases with necrotic lesions: tan spot, downy mildew, gummy stem blight, and the foliar disease complex of tomato. The coefficients of determination were slightly lower for the rust and powdery mildew images $\left(R^{2} \geq 0.79\right.$; Table 2). Slopes ranged from 0.65 to 0.99 and were not significant from $1(P \geq 0.05)$ in all disease datasets with the exception of lilac powdery mildew $(P<0.001)$. $Y$-intercepts ranged from -1.67 to 16.63 and were significantly different from zero for all disease datasets $(P<0.001 ;$ Table 2$)$. Concordance correlation coefficients across all datasets were at least 0.959 and in four of the datasets greater than 0.99 (Table 2).

Precision. Coefficients of variation for repeated estimates of disease severity using Leaf Doctor were low for all diseases (Table 3). The highest coefficients of variation were obtained for the rust and powdery mildew images. These coefficients were on average 3.5 times higher than for the other diseases, which had necrotic lesions with clear delineations between healthy and symptomatic tissue. There was a significant negative relationship between the

Table 2. Lin's concordance correlation coefficient and regression statistics and model parameters for linear regression analyses between disease severity assessments from Assess and Leaf Doctor within each of the six disease datasets $(n=50)$

\begin{tabular}{lcccccc}
\hline Disease (host) & $\boldsymbol{C}_{\mathbf{b}}{ }^{\mathbf{a}}$ & $\boldsymbol{R}^{\mathbf{2 b}}$ & Intercept & Slope & SEEy & $\boldsymbol{P}=$ \\
\hline Tan spot (lima bean) & 0.997 & 0.94 & -0.11 & 0.95 & 2.65 & $<0.001$ \\
Downy mildew (collard) & 0.994 & 0.96 & 1.54 & 0.99 & 4.05 & $<0.001$ \\
Rust (mallow) & 0.959 & 0.88 & 1.68 & 0.73 & 4.93 & $<0.001$ \\
Powdery mildew (lilac) & 0.963 & 0.79 & 16.63 & 0.65 & 12.22 & $<0.001$ \\
Foliar disease complex & 0.990 & 0.95 & -1.67 & 0.98 & 4.22 & $<0.001$ \\
$\quad$ (tomato) & & & & & & \\
$\quad$ Gummy stem blight & 0.998 & 0.98 & 0.83 & 0.94 & 3.60 & $<0.001$ \\
$\quad$ (watermelon) & & & & & &
\end{tabular}

a Lin's concordance correlation coefficient.

b Coefficient of determination.

c Standard error of the estimate of $y$.

Table 3. Mean, minimum, and maximum coefficient of variation of disease severity estimates made by Leaf Doctor on 10 randomly selected leaves within each of the disease databases. Each image was imported separately into Leaf Doctor on five separate occasions for assessment of disease severity (\%)

\begin{tabular}{lccc}
\hline & \multicolumn{3}{c}{ Coefficient of variation (\%) } \\
\cline { 2 - 4 } Disease (host) & Mean & Minimum & Maximum \\
\hline Tan spot (lima bean) & 2.26 & 0.57 & 6.96 \\
Downy mildew (collard) & 0.51 & 0.05 & 2.74 \\
Rust (mallow) & 14.10 & 3.17 & 37.50 \\
Powdery mildew (lilac) & 13.16 & 6.90 & 38.61 \\
Foliar disease complex (tomato) & 7.74 & 2.65 & 13.01 \\
Gummy stem blight (watermelon) & 5.27 & 2.47 & 9.28 \\
\hline
\end{tabular}


coefficients of variation and mean disease severity estimated by Leaf Doctor in all diseases with the exception of the foliar disease complex in tomato $(P=0.683 ;$ Fig. 4). Significant linear relationships were fitted for those in mallow rust $\left(R^{2}=0.72 ; P=0.002\right)$, lilac powdery mildew $\left(R^{2}=0.77 ; P<0.001\right)$, and gummy stem blight $\left(R^{2}=0.56\right.$; $P=0.013)$. Significant relationships were also observed between the coefficient of variation and mean disease severity for tan spot of lima bean $(P=0.003)$ and downy mildew of collards $(P=0.024)$. These relationships were best described by negative exponential and second-order polynomial equations (Fig. 4).

Processing speed. Time required to process images through Leaf Doctor was substantially less than Assess (Table 4). On average, image processing time using Assess was between 3.1 and 6.8 times greater than when analyzing the same images with Leaf Doctor. The average time to process an individual image through Leaf Doctor varied from 32.8 to $71.6 \mathrm{~s}$ for the foliar disease complex on tomato
A

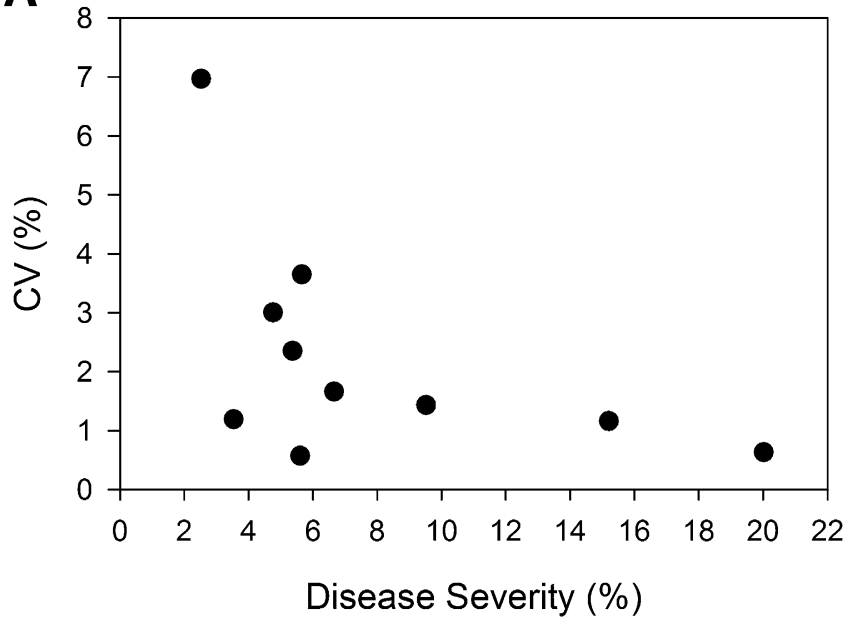

C

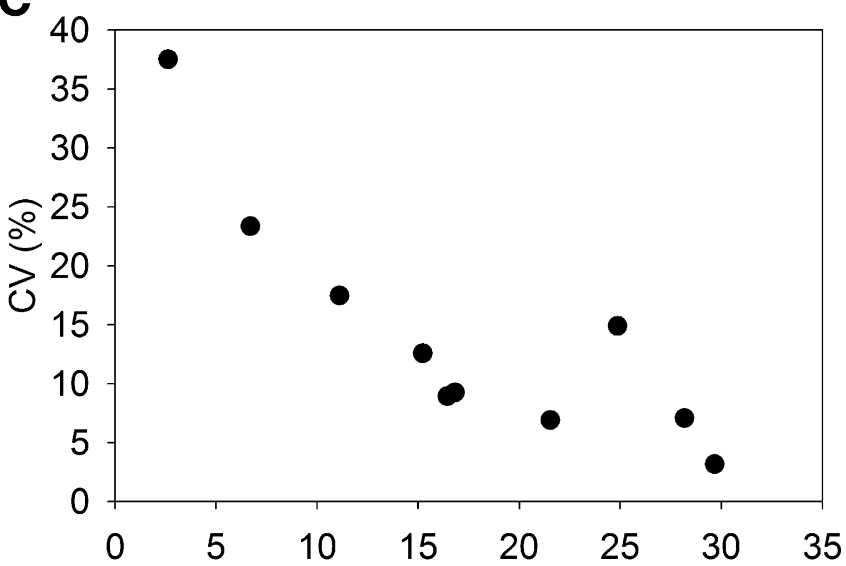

E

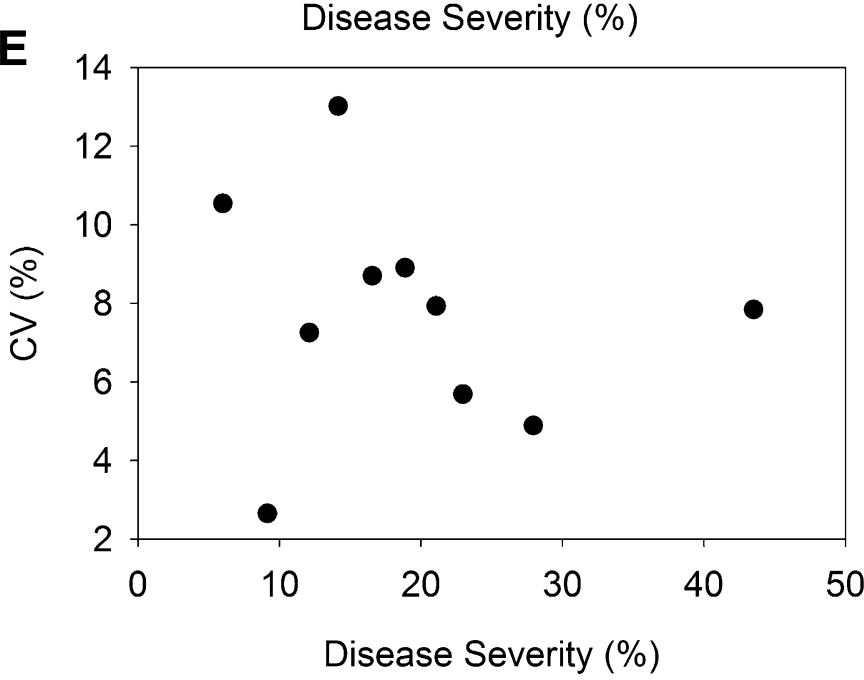

B

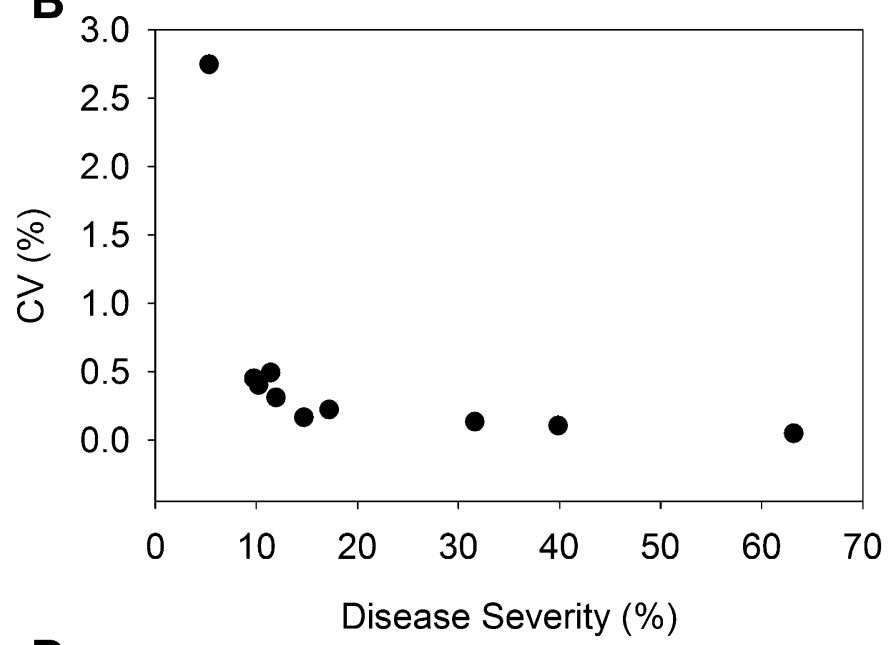

D

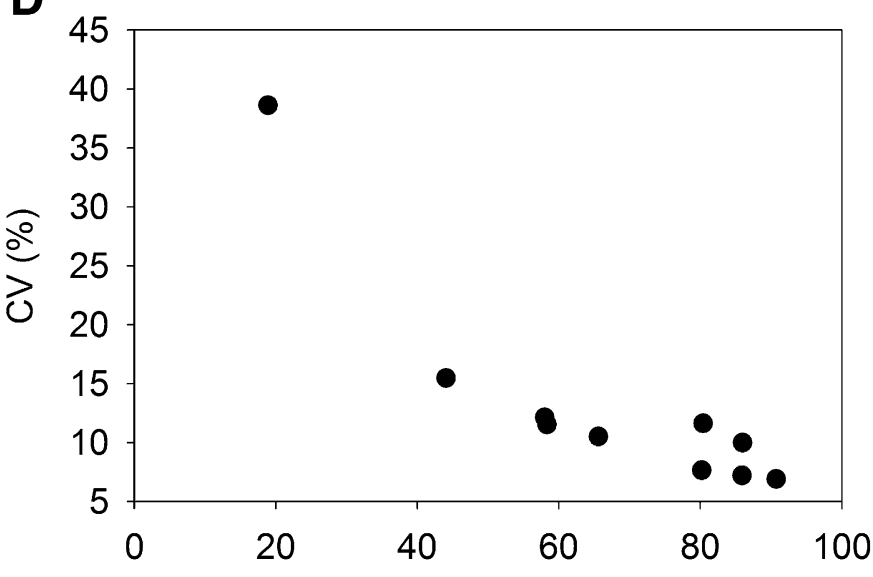

$\mathbf{F}$

Disease Severity (\%)

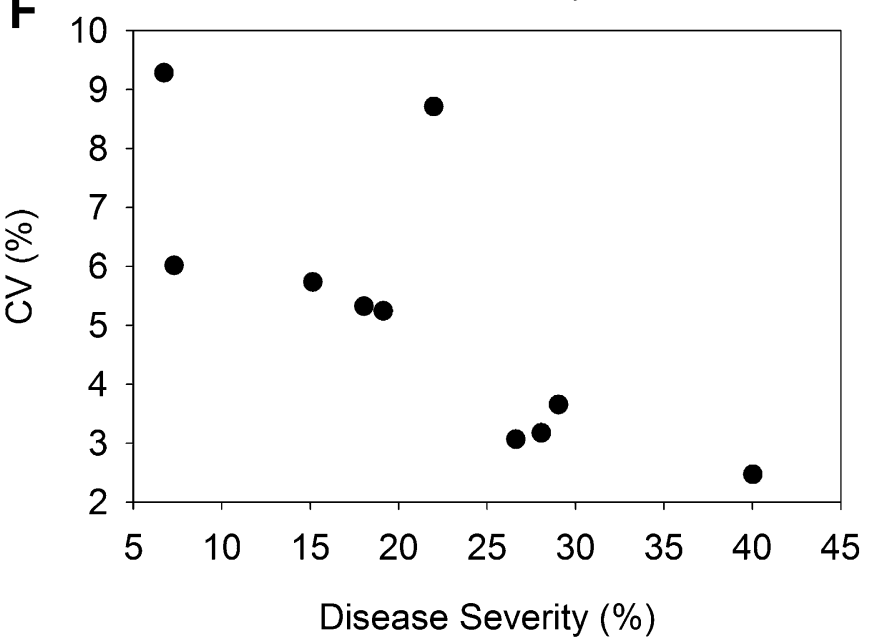

Fig. 4. Relationships between the coefficient of variation (\%) and disease severity (\%) estimates made using Leaf Doctor. Five estimates of disease severity were made on 10 individual randomly selected leaf images for tan spot of lima bean (A), downy mildew of collards (B), mallow rust (C), lilac powdery mildew (D), foliar disease in tomato (E), and gummy stem blight of watermelon (F). 
and powdery mildew on lilac, respectively. The standard deviation of processing time was also substantially higher when using Assess (Table 4).

Creation of a standard area diagram using Leaf Doctor. Beach morning-glory leaves with Cercospora leaf spot abscised at or before $23 \%$ disease, which was regarded as maximum severity for this disease. Images of representative diseased leaves in each of six disease severity assessment categories were selected to approximate the midpoint of the ranges (Fig. 5). The time to construct the standard area diagram was $50 \mathrm{~min}$.

\section{Discussion}

Leaf Doctor is a new tool for the semiautomatic assessment of disease severity for individual leaves or other sampling units. Evaluation of the Leaf Doctor algorithm for a range of lesion types, leaf shapes, and disease intensities demonstrated that this application provided estimates that were highly accurate when compared with estimates from a commonly used discipline standard, precise, and robust. Linear relationships were significant among estimates for all diseases studied with approximately $1: 1$ relationships and high coefficients of determination $\left(R^{2} \geq 0.79\right)$ and standard errors of the $y$ estimate, indicating a low degree of error associated with the

Table 4. Mean and standard deviation in processing times for calculating disease severity across 10 randomly selected leaves within each of the disease databases using Assess and Leaf Doctor

\begin{tabular}{lcc}
\hline & \multicolumn{2}{c}{$\begin{array}{c}\text { Mean (standard deviation) } \\
\text { processing times (s) }\end{array}$} \\
\cline { 2 - 3 } Disease (host) & Assess & Leaf Doctor \\
\hline Tan spot (lima bean) & $211.6(20.5)$ & $50.7(11.1)$ \\
Downy mildew (collard) & $238.5(22.3)$ & $34.9(5.7)$ \\
Rust (mallow) & $241.4(12.6)$ & $42.0(5.4)$ \\
Powdery mildew (lilac) & $219.2(24.8)$ & $71.6(14.6)$ \\
Foliar disease complex (tomato) & $174.8(16.5)$ & $32.8(5.1)$ \\
Gummy stem blight (watermelon) & $297.0(18.3)$ & $49.7(7.3)$ \\
\hline
\end{tabular}

predicted $y$-value. The accuracy of estimates by Leaf Doctor was relatively higher in diseases featuring necrotic lesions $\left(R^{2} \geq 0.94\right.$; SEE $y \leq 4.93)$ with almost perfect concordance correlation coefficients $\left(C_{\mathrm{b}} \geq 0.99\right)$. Coefficients of determination were slightly lower with correspondingly higher standard errors for estimates of disease severity in mallow rust and powdery mildew of lilac. This was likely due to the higher contrast in color between healthy and diseased sections of leaves in diseases with necrotic symptoms. Powdery mildew of lilac was the only dataset where a systematic and constant bias was identified in disease severity assessments made by Leaf Doctor in comparison with Assess with a slope and intercepts deviating significantly from one and zero, respectively. A reduction in accuracy and robustness of algorithms used for disease severity estimation has also been reported in powdery mildew and other diseases where contrast is compromised (Barbedo 2014). Although coefficients of variation in most datasets were minimal among the diseases included in this study, significant negative linear or nonlinear relationships between the coefficient of variation and mean disease severities were observed. Similar phenomena have also been reported in the accuracy of manual disease severity estimations conducted by individual raters (Barbedo 2014; Sherwood et al. 1983).

In addition to the statistical comparisons to quantify the accuracy, precision, and robustness of Leaf Doctor, faster processing times than Assess were also a substantial advantage. Reported processing times for Assess are approximately $20 \mathrm{~s}$ (Barbedo 2014). In this

Table 5. Comparative advantages and disadvantages of Leaf Doctor and Assess for quantification of disease severity

\begin{tabular}{lll}
\hline Factor & \multicolumn{1}{c}{ Leaf Doctor } & \multicolumn{1}{c}{ Assess } \\
\hline Cost (USD) & 0 (free download) & $\sim 780$ \\
East of use & Easy & Difficult \\
Portability & High & Low \\
Built-in camera & Yes & No \\
Accuracy & High & High \\
Precision & High & Not tested in this study \\
\hline
\end{tabular}

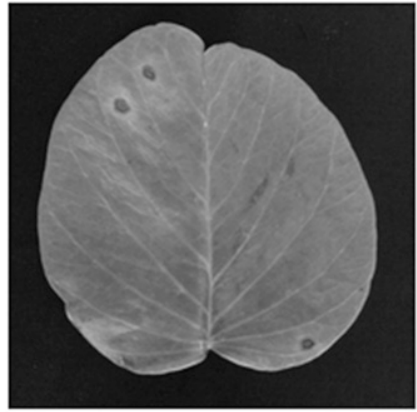

1

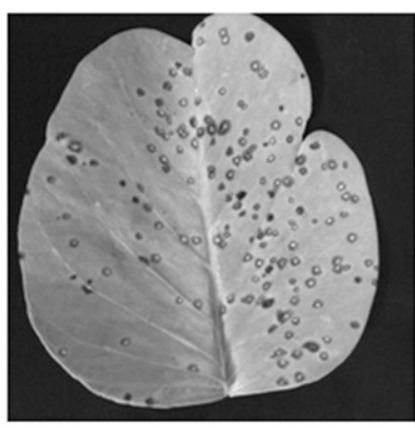

4

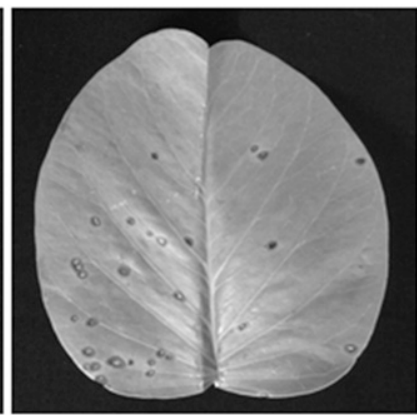

2

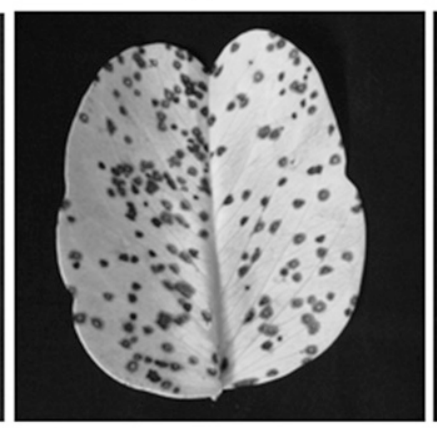

5

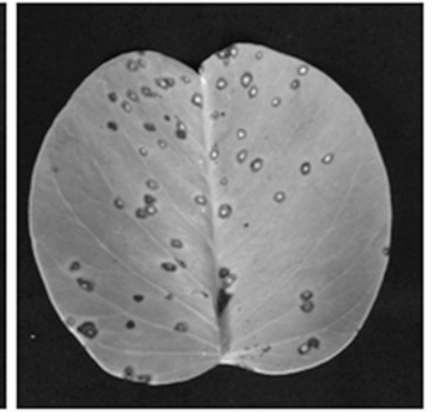

3

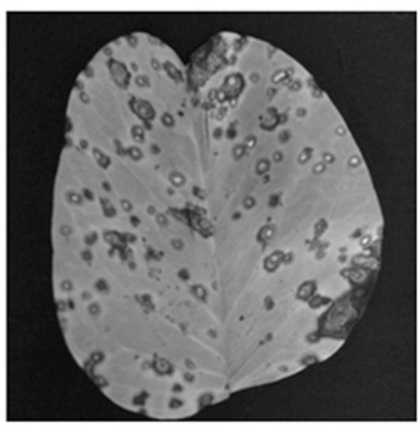

Maximum

Fig. 5. Standard area diagram for Cercospora leaf spot of beach morning-glory constructed using Leaf Doctor according to a six-category logarithmic scale $(1=0$ to $1 \% ; 2=1$ to $3 \% ; 3=3$ to $6 \% ; 4=6$ to $12 \% ; 5=12$ to $25 \%$; and $6=$ maximum observed). Actual disease severities displayed here are $0.34 \%(1) ; 1.27 \%(2) ; 5.09 \%(3) ; 9.63 \%(4) ; 17.4 \%(5) ;$ and $22.56 \%(6)$ 
study, the average time required to process an image through Assess was approximately $300 \mathrm{~s}$. The user processing images was experienced with both programs. However, processing by Leaf Doctor was substantially faster than Assess. This was partly due to Leaf Doctor's ease of use. All aspects of image analysis, including uploading the image and the ability to select multiple areas of the image to be designated healthy were markedly faster. The ability to rapidly select multiple areas with Leaf Doctor and designate or de-designate them as a binary category ("healthy") renders considerable benefits when compared with other image analysis systems.

The ability to select up to eight colors for "healthy" tissue contributes directly to the increased accuracy and precision of Leaf Doctor. The application also allows a user to quantify disease severity in the presence or absence of different symptom colors on a sampling unit. For example, for diseases where symptoms include two contrasting colors, such as necrosis (e.g., dark hues) and chlorosis (e.g., yellowish hues), users of Leaf Doctor can quantify disease severity by including the area affected by both symptoms, or by excluding either one or the other symptom from analysis. This system also allows users to select leaf veins that often have a different spectral signature from those sections of the leaf that are green and healthy or are diseased. The inability of other image analysis systems to select leaf veins as healthy tissue is often a substantial source of estimate error (Barbedo 2014).

Another use of Leaf Doctor is the rapid construction of standard area diagrams from actual pathosystems based on estimates of disease made by trained raters. The benefits of standard area diagrams include improved accuracy and precision of intrarater estimates and better interrater reproducibility (Braido et al. 2014; Nutter et al. 1993). However, all or most standard area diagrams are prepared using stylized representations of the sampling unit (e.g., fruit or leaf) and the varying levels of disease severity are often represented by a dark colored area that is unlikely to accurately portray actual symptoms. Leaf Doctor's "slider" function can create realistic simulations of disease intensity that account for the subtle nuances of plant disease symptoms (Fig. 1). This includes whether lesions are most likely to begin at leaf margins, or coalesce from initial lesions that may be uniformly distributed across a leaf surface.

Other potential advantages and disadvantages of using Leaf Doctor for image analysis are compared and contrasted in Table 5. Further, the availability of Leaf Doctor on a mobile platform allows portability and flexibility for on-site collection of data. In contrast, Assess requires a desktop computer, or if the program is installed on a laptop, does not offer equivalent functionality, as laptops lack cameras that can readily capture images. For Assess, therefore, images must be imported from an external camera or other device. In addition, Assess is not compatible with iOS nor does it function on any smartphone or mobile device. The relative ease of use and rapid processing times intrinsic to Leaf Doctor allow its use by those without highly developed skills in computer programming or image analysis. These special skills are usually essential for the proper implementation of other image analysis systems, with the exception of Assess.

A potential limitation of Leaf Doctor is the required black background for image analysis. If disease symptoms display solid black hues, they may not be sufficiently distinguished from the background and the respective pixels not quantified as diseased. Furthermore, destructive sampling of leaves for photography may disrupt epidemics. Programming improvements to the application could allow photography of diseased leaves in situ, automatic recognition of sampling unit margins, and creation of a black background. This capacity would allow for repeated measurements of a sampling unit of interest. However, destructive sampling is not required if the user applies the required background color using computer software for image editing.

Leaf Doctor is not limited to analysis of diseased leaves or even phytopathometry. For example, a user can quantify the extent of visible damage to wood by termites, or analyze an aerial image of a field of plants to quantify disease incidence in a population. A user could also quantify cloud cover in a photograph of the sky. A programmer could use the algorithm of Leaf Doctor to create a new method of pixel-based analysis of spatial patterns for diseases on plant tissues, or in fields, forests, or landscapes.
Leaf Doctor is free to download from iTunes to an Apple mobile platform and therefore may be used on an iPad, iPhone, or iPod touch. The application is optimized for iPhone 5. The source code for Leaf Doctor is available free of charge by contacting the corresponding author.

\section{Acknowledgments}

Funding for the development of the Leaf Doctor application was provided by the United States Department of Agriculture, National Institute of Food and Agriculture (USDA-NIFA) Hatch project managed by the College of Tropical Agriculture and Human Resources, University of Hawaii at Manoa. The testing of Leaf Doctor was supported by the USDA-NIFA Hatch project NYG-625424, managed by The New York Agricultural Experiment Station, Cornell University, Geneva. The authors acknowledge Iulian Corcoja (AQUASoft, Romania) and Dragos Ione (Adelante Consulting Inc., Canada) for app coding and design. Thanks to Christine Smart, Cornell University and Fred Brooks, University of Hawaii at Manoa, for their constructive comments on earlier drafts of this manuscript.

\section{Literature Cited}

Abramoff, M., Magalhães, P., and Ram, S. 2004. Image Processing with ImageJ. Biophotonics Int. 11:36-42.

Barbedo, J. G. A. 2014. An automatic method to detect and measure leaf disease symptoms using digital image processing. Plant Dis. 98:1709-1716.

Bardsley, S. J., and Ngugi, H. K. 2013. Reliability and accuracy of visual methods used to quantify severity of foliar bacterial spot symptoms on peach and nectarine. Plant Pathol. 62:460-474.

Bock, C. H., Parker, P. E., Cook, A. Z., and Gottwald, T. R. 2008. Visual rating and the use of image analysis for assessing different symptoms of citrus canker on grapefruit leaves. Plant Dis. 92:530-541.

Braido, R., Goncalves-Zuliani, A. M. H., Janeiro, V., Carvalho, S. A., Belasque Junior, J., Bock, C. H., and Nunes, W. M. C. 2014. Development and validation of standard area diagrams as assessment aids for estimating the severity of citrus canker on unripe oranges. Plant Dis. 98:1543-1550.

Gaunt, R. E. 1995. New technologies in disease measurement and yield loss appraisal. Can. J. Plant Pathol. 17:185-189.

Horsfall, J. G., and Barratt, R. W. 1945. An improved grading system for measuring plant disease. Phytopathology 35:655 (Abstr.).

James, W. C. 1971. A Manual of Assessment Keys for Plant Diseases. Can. Dpt Agric. Publication no. 1458

Kranz, J. 1988. Measuring plant disease. Pages 35-50 in: Experimental Techniques in Plant Disease Epidemiology. J. Kranz and J. Rotem, eds. Springer-Verlag, New York.

Kwack, M. S., Kim, E. N., Lee, H., Kim, J.-W., Chun, S.-C., and Kim, K. D. 2005. Digital image analysis to measure lesion area of cucumber anthracnose by Colletotrichum orbiculare. J. Gen. Plant Pathol. 71:418-421.

Lamari, L. 2002. ASSESS: Image Analysis Software for Plant Disease Quantification. American Phytopathological Society, St. Paul, MN.

Lin, L. I.-K. 1989. A concordance correlation coefficient to evaluate reproducibility. Biometrics 45:255-268.

Lindow, S. E., and Webb, R. 1983. Quantification of foliar plant disease symptoms by microcomputer-digitized video image analysis. Phytopathology 73:520-524

Madden, L. V., Hughes, G., and van den Bosch, F. 2007. The Study of Plant Disease Epidemics. The American Phytopathological Society, St. Paul, MN.

Nilsson, H.-E. 1995. Remote sensing and image analysis in plant pathology. Annu. Rev. Phytopathol. 33:489-527.

Nutter, F. W., Jr., Gleason, M. L., Jenco, J. H., and Christians, N. C. 1993. Assessing the accuracy, intra-rater repeatability, and inter-rater reliability of disease assessment systems. Phytopathology 83:806-812.

Nutter, F. W., Jr., and Schultz, P. M. 1995. Improving the accuracy and precision of disease assessments: selection of methods and use of computer-aided training programs. Can. J. Plant Pathol. 17:174-184.

Nutter, F. W., Jr., Teng, P. S., and Shokes, F. M. 1991. Disease assessment terms and concepts. Plant Dis. 75:1187-1188.

Patil, S. B., and Bodhe, S. K. 2011. Leaf disease severity measurement using image processing. Int. J. Eng. Technol. 3:297-301.

Price, T. V., Gross, R., Wey, J. H., and Osborne, C. F. 1993. A comparison of visual and digital image-processing methods in quantifying the severity of coffee lea rust (Hemileia vastatrix). Aust. J. Exp. Agric. 33:97-101.

Sherwood, R. T., Berg, C. C., Hoover, M. R., and Zeiders, K. E. 1983. Illusions in visual assessment of Stagonospora leaf spot of orchard grass. Phytopathology 73:173-177.

Spolti, P., Schneider, L., Sanhueza, R. M. V., Batzer, J. C., Gleason, M. L., and Medeiros Del Ponte, E. 2011. Improving sooty blotch and flyspeck estimation on apple fruit with the aid of standard area diagrams. Eur. J. Plant Pathol. 129:21-29.

Stevens, F. L. 1925. Hawaiian fungi. Pages 1-10 in: Bernice P. Bishop Museum Bulletin 19. Honolulu, HI.

Tucker, C. C., and Chakraborty, S. 1997. Quantitative assessment of lesion characteristics and disease severity using digital image processing. J. Phytopathol. 145:273-278.

Wijekoon, C. P., Goodwin, P. H., and Hsiang, T. 2008. Quantifying fungal infection of plant leaves by digital image analysis using Scion Image software. J. Microbiol. Methods 74:94-101. 